Article

\title{
Application of WOA-SVR in Seed Vigor of High-Voltage Electric Field Treatment on Aged Cotton (Gossypium spp.) Seeds
}

\author{
Zhanhua Song ${ }^{1,2,3}$, Junxiang Ma ${ }^{1,4}$, Qian Peng ${ }^{1}$, Baoji Liu ${ }^{1}$, Fade Li $^{1,2}{ }^{\text {, Xiangyun Sun }}{ }^{1}$, Mochen Liu ${ }^{1,2, *}$ \\ and Yinfa Yan $1,2, * \mathbb{D}$
}

check for updates

Citation: Song, Z.; Ma, J.; Peng, Q.; Liu, B.; Li, F.; Sun, X.; Liu, M.; Yan, Y. Application of WOA-SVR in Seed Vigor of High-Voltage Electric Field Treatment on Aged Cotton (Gossypium spp.) Seeds. Agronomy 2022, 12, 88. https://doi.org/ 10.3390/agronomy 12010088

Academic Editor: Petr Smýkal

Received: 25 November 2021

Accepted: 27 December 2021

Published: 30 December 2021

Publisher's Note: MDPI stays neutral with regard to jurisdictional claims in published maps and institutional affiliations.

Copyright: (C) 2021 by the authors. Licensee MDPI, Basel, Switzerland. This article is an open access article distributed under the terms and conditions of the Creative Commons Attribution (CC BY) license (https:// creativecommons.org/licenses/by/ $4.0 /)$.
1 School of Mechanical and Electronic Engineering, Shandong Agricultural University, Taian 271018, China; songzh@sdau.edu.cn (Z.S.); sdmjxy@163.com (J.M.); 15264892120@163.com (Q.P.); liubaoji94@163.com (B.L.); lifade@sdau.edu.cn (F.L.); yun230828@163.com (X.S.)

2 Shandong Key Laboratory of Horticultural Machinery and Equipment, Taian 271018, China

3 Shandong Provincial Engineering Laboratory of Intelligent Agricultural Equipment, Taian 271018, China

4 Shandong Weifang Bank Co., Ltd., Weifang 261000, China

* Correspondence: liu_mochen@126.com (M.L.); yanyinfa@sdau.edu.cn (Y.Y.)

\begin{abstract}
When seeds are treated with a high-voltage electric field (HVEF) to improve seed vigor due to the large differences in the biological electromagnetic effects on different types of seeds, the methods of variance analysis and regression analysis based on data statistics are generally used to construct the optimal electric field dose prediction model; however, the generalization performance of the prediction model tends to be poor. To solve this problem, the electric intensity, frequency and treatment time were taken as the input variables for hybrid support vector regression (SVR) analysis to establish the prediction model of the seed comprehensive germination index. The whale optimization algorithm (WOA) was used to optimize the kernel parameters of the SVR. The optimized hybrid WOA-SVR model predicted the optimal comprehensive germination index of aged cotton (Gossypium spp.) seeds to be 329, the optimal HVEF dosage was $3.64 \mathrm{kV} / \mathrm{cm} \times 99 \mathrm{~s}$, and the frequency was $1.4 \mathrm{~Hz}$. The aged cotton seeds were treated with the optimal HVEF and the germination test was carried out. Compared with the check (CK), the comprehensive germination index of seeds increased by $48 \%$. The research results provided a new method and new idea for the optimal design of parameters for seed treatment with HVEF.
\end{abstract}

Keywords: seed vigor; WOA-SVR; biological electromagnetic effects; model prediction; HVEF

\section{Introduction}

Seed vigor is an important index for evaluating seed quality [1]. High-vigor seeds have significant growth advantages and high yield potential, which is the primary condition for high efficiency and high yield in modern agriculture [2]. Studies have shown that proper high-voltage electric field (HVEF) treatment on seeds could change the permeability of seed cell membranes [3-5], increase the activity of intracellular enzymes [6], accelerate cell mitosis and radicle development, promote cell metabolism [7-9], and significantly improve seed vigor [10]. Xu et al. used the corona field to treat rice seeds and found that the treated seeds not only improved the germination potential, germination rate, germination index and other indexes, but also the germination peak was about 1 day earlier than the control [11,12]. Lynikieney et al. had found that the corona field treatment could advance the germination peak of vegetable seeds by 2 to 3 days earlier [13]. Marček et al. treated wheat seeds with high voltage discharge, found that high voltage pretreatment of wheat seeds improved the root length, seedling length, germination potential and germination index of seeds, and increased the electrical conductivity of water medium [14]. In addition, some studies have shown that HVEF treatment on seeds could improve the cold stress [15], 
drought stress [16], and salinity stress $[17,18]$ in seeds. Bormashenko et al. studied the effect of cold radiofrequency plasma on the water absorption of the outer epidermis of beans, and found that the plasma-treated beans accelerated the water absorption rate of the seeds' outer epidermis, increased the germination rate of the seeds, and made the seeds outer epidermis more hydrophilic [19]. Wang et al. studied the effect on millet seeds treated with corona discharge field, and the results showed that the vigor index of millet seeds treated with corona discharge field was significantly higher than that of the control. By measuring the conductivity of seed leachates, it was found that corona field treatment of seeds could effectively promote the integrity of cell membrane during seed germination [20]. Patwardhan et al. used HVEF to treat tomato seeds and carried out seed germination experiments, and the seed germination data were subjected to regression analysis and variance test. They found that the treatment effect was the best when the treatment time was $20 \mathrm{~s}$ and the electric field intensity was $200 \mathrm{kV} / \mathrm{m}$, compared with the control; moreover, the seed germination rate of the HVEF treatment increased from $76 \%$ to $100 \%$, and made a very significant difference [21]. Using data envelopment analysis and non-parametric comprehensive analysis method, Bao et al. analyzed the comprehensive effects of different electric field intensities and treatment times on the biological effects of wheat seeds, and revealed the internal relationship in seedling morphological indexes between the different electric field treatments and the control [22]. Yan et al. used low-frequency and pulsed HVEF to treat aged cotton seeds, designed orthogonal experiments and optimized the electric field treatment conditions with response surface modeling. Compared with the control, germination potential, germination rate, germination index and vigor index made an extremely significant difference [23]. Chen et al. established a mathematical model between electric field intensity, treatment time and comprehensive indexes of millet seed germination by using the combination of quadratic general rotation combination design and principal component analysis. The optimized electric field conditions significantly affected the germination vigor of millet seeds [24]. Different electric field intensities, frequencies and treatment times have different effects on seed vigor. Accurately predicting the changing trend of seed vigor under different HVEF treatment conditions, establishing seed vigor and HVEF parameter model, and predicting the optimal dose of electric field (electric field dose $=$ electric field intensity $\times$ treatment time) is of great significance for improving seed vigor and quality.

In recent years, with the rapid development of machine learning, the use of machine learning algorithms instead of traditional mathematical statistical methods for model construction is emerging $[25,26]$. Support vector regression (SVR) is a machine learning method based on statistical learning theory. Its key idea is to map the sample space to the high-dimensional feature space through the "kernel function mapping", and use the risk minimization principle to construct decision function, which is suitable for solving nonlinear problems with a small number of samples and high dimensionality [27]. SVR model parameters, such as penalty factor $c$ and kernel function parameter $g$, have a significant impact on the performance of the model and are difficult to select; parameter optimization algorithms are often introduced in the modeling process to optimize SVR model parameters [28].

This paper conducted HVEF treatment on aged cotton seeds and germination experiments, then calculated the germination potential, germination rate, vigor index, germination index and comprehensive germination index of each group of experimental seeds. The small-sample data of the orthogonal experiment of HVEF treatment on seeds was used as the training set. SVR was used to establish a seed vigor prediction model for HVEF treatment, and the whale optimization algorithm (WOA) [29] was used to intelligently optimize the parameters of the SVR model, which improved the accuracy of the SVR regression model of seed vigor. This study provided a new research idea for seed vigor prediction based on small-sample germination experimental data of HVEF treatment on seed and SVR, and provided a new method for selecting the optimal electric field dose for HVEF treatment on seeds. 


\section{Materials and Methods}

\subsection{Raw Material}

The aged cotton (Gossypium spp.) seeds (Xinmian2, long staple cotton, $6 \pm 0.6 \%$ moisture content) were produced in 2016 and provided by Agronomy College of Shandong Agricultural University, Shandong, China. The seeds had been stored in a dry place at room temperature before the experiments. Undamaged and plump seeds were manually selected for HVEF treatment and germination experiments.

\subsection{Experiment Instruments}

The HVEF seed treatment device was mainly composed of a pulsed high-voltage power supply (self-developed by the School of Mechanical and Electrical Engineering, Shandong Agricultural University), arc-shaped prick electrodes, high-voltage probe (Tektronix P6015A, Tektronix Inc., Beaverton, OR, USA), oscilloscopes (Tektronix TDS1012B-SC, Tektronix (China) Inc., Shanghai, China) and grounded flat electrode. The schematic diagram of the electric field seed treatment device is shown in Figure 1. Each piece of prick electrode with a thickness of $0.6 \mathrm{~mm}$ had 24 arc-shaped tips with a radius of $4.5 \mathrm{~mm}$. The angle between the two tangents of the arc was $35^{\circ}$. The seed treatment device was installed with 10 prick electrodes, the distance between each two electrodes was $24 \mathrm{~mm}$, the gap between the tip of the arc-shaped electrode and the plate electrode was $50 \mathrm{~mm}$, and the size of the plate electrode was $240 \mathrm{~mm} \times 400 \mathrm{~mm}$. The specific shape can be found in the references of our laboratory [11,12,30].

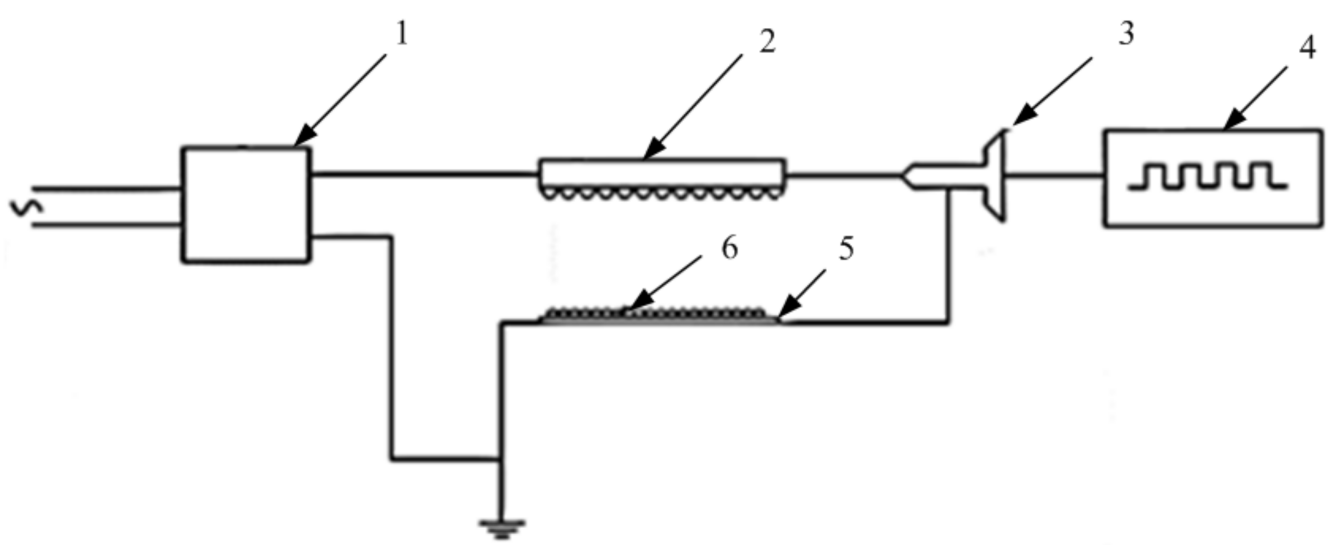

Figure 1. HVEF seed treatment device. 1. Pulsed high-voltage power supply. 2. Arc-shaped prick electrodes. 3. High voltage probe. 4. Oscilloscope. 5. Grounded plate electrode. 6. Cotton seeds.

\subsection{Experiment Methods}

\subsubsection{Pulsed Electric Field Treatment Experiment}

To eliminate the influence of temperature and humidity on the energy release of HVEF-treated seeds, electrical treatment in an air-conditioned room with size of $2 \mathrm{~m} \times 2 \mathrm{~m}$ was built in our laboratory, the room temperature was automatically adjusted by the air conditioner (Gree KFR-26GW, Gree Electric Appliances Inc. of Zhuhai, China) and the humidity was regulated by the intelligent humidifier (Philips HU4803, Philips (China) Investment Co., Ltd., Shanghai, China).

The HVEF treatment experiment was carried out in the air-conditioned room with a temperature of $25^{\circ} \mathrm{C} \pm 2{ }^{\circ} \mathrm{C}$ and relative humidity of $30 \% \pm 3 \%$, the distance between the arc-shaped prick electrode and the grounded plate electrode was $5 \mathrm{~cm}$, and the output voltage of the pulsed high-voltage power supply formed an electric field for treating seeds between the two plates. The cotton seeds were evenly spread on the grounded plate electrode. According to the preliminary experiment of HVEF treatment on aged cotton seeds, the HVEF intensity was in the range of $2.4-4 \mathrm{kV} / \mathrm{cm}$, the pulse frequency was $1-50 \mathrm{~Hz}$, and the treatment time was 20-100 s [24]. To reduce the influence of the 
decreasing electromagnetic effect on the germination of seeds, the seeds were planted in the germination bed within $12 \mathrm{~h}$ after being treated with HVEF [31].

\subsubsection{Seed Germination Experiment}

The cotton seeds treated by the pulsed HVEF and the control were immersed and disinfected with $60 \mathrm{mg} / \mathrm{L}$ electrolyzed functional water for $10 \mathrm{~min}$, then rinsed with deionized water for 3 times and dried with absorbent paper; the seeds were then planted evenly and neatly in the germination box with sand bed. To prevent evaporation of water from the germination box, the box was covered with a lid before the cotton seeds germinated. One hundred (100) cotton seeds were randomly picked as one germination repetition, and each treatment group was subjected to 5 repetition experiments. The seed germination experiments were carried out in the germination room of the State Key Laboratory of Crop Biology, Shandong Agricultural University. The germination room, at $30{ }^{\circ} \mathrm{C} \pm 2{ }^{\circ} \mathrm{C}$ and $100 \% \mathrm{RH}$, was set at $12 \mathrm{~h}$ light and $12 \mathrm{~h}$ dark alternately. During the experiment, the moldy or rotten seeds were cleaned up and removed in a timely manner. On the 3rd day of germination, some cotton seeds with higher vigor began to germinate, and some of the higher seedlings were close to the germination box lid. To prevent the lid from affecting the growth of cotton seedlings, the germination box lid was removed in time. After removing the lid, the water requirement of the seedlings was increased and the water evaporation of the sand bed was increased, so the seedlings were watered by spraying water once or twice a day to keep the sand bed moist. From the 4 th day onward, the number of germinated seeds in each box was recorded every day, and the seedlings were washed at the end of the germination on the 12th day. Then, seedling length was measured, and the average seedling length for each replicate was calculated. Finally, the germination potential, germination rate, germination index, vigor index and comprehensive germination index of each replicate were calculated according to Equations (1)-(7) as follows:

$$
\begin{gathered}
G E=G_{4} / 100 \\
G P=G_{12} / 100 \\
G I=\sum G_{t} / D_{t} \\
V I=G I \cdot S \\
P V=G_{t} / T \\
M D G=G P / G_{D} \\
W=[V I+P V \cdot M D G+(B-M L T I)] / 3
\end{gathered}
$$

where GE is germination potential, $\% ; G_{4}$ is the number of cotton seeds germinated at the 4 th day; GP is germination rate, $\%$; $G_{12}$ is the number of cotton seeds germinated at the 12th germination day; GI is germination index; $D_{t}$ is the days of seeds germination; $G_{t}$ is the number of seed germination per day corresponding to $D_{t} ; V I$ is vigor index of seeds; $S$ is the average weight of a seedling, $g$; $P V$ is germination peak; $G_{t}$ is germination rate at the germination peak; $T$ is the days of the germination peak; $M D G$ is average germination rate per day; $G_{D}$ is germination days; $B$ is the maximum germination days; MLTI is average germination days; and $W$ is comprehensive germination index.

\subsection{Creating Cotton Seeds Germination Data Set}

To ensure that the small sample data of the multi-factor experiment could represent the comprehensive experiment of seed germination, the orthogonal experiment was adopted to design the seed germination experiment, and the orthogonal experiment data of aged cotton seed germination was used as the raw sample data set for SVR regression model analysis.

Taking HVEF intensity $3 \mathrm{kV} / \mathrm{cm}$, frequency $10 \mathrm{~Hz}$, and treatment time $60 \mathrm{~s}$ as the center point, using Minitab software to design cotton seed germination orthogonal test, the orthogonal test table with three factors and five levels is shown in Table 1. 
Table 1. Orthogonal test table.

\begin{tabular}{cccc}
\hline Level & \multicolumn{3}{c}{ Factor } \\
\cline { 2 - 4 } & $\begin{array}{c}\text { HVEF Intensity } \\
(\mathbf{k V / c m})\end{array}$ & $\begin{array}{c}\text { Frequency } \\
\mathbf{( H z )}\end{array}$ & $\begin{array}{c}\text { Treatment Time } \\
\mathbf{( s )}\end{array}$ \\
\hline 1 & 2.4 & 1 & 20 \\
2 & 2.6 & 5 & 40 \\
3 & 3 & 10 & 60 \\
4 & 3.4 & 20 & 80 \\
5 & 3.6 & 50 & 100 \\
\hline
\end{tabular}

\subsubsection{Method of Establishing Seed Vigor Model}

The HVEF intensity, frequency and treatment time of orthogonal test data of aged cotton seed germination were used as the raw sample data, which were normalized for establishing the SVR model, and the comprehensive germination index was the output of SVR model. The WOA was used to optimize the SVR model parameters $c$ (penalty factor) and $g$ (kernel function parameter).

\subsubsection{Kernel Function of SVR}

Compared with a single kernel function, using multiple kernel functions can effectively improve the accuracy of the regression prediction model. The Gaussian kernel function is a local kernel function with weak learning ability. On the other hand, the polynomial kernel function is a global kernel function, with weak generalization ability but has strong learning ability $[32,33]$.

In this paper, a hybrid kernel function SVR with Gaussian kernel and polynomial kernel superimposed was used, which not only had global generalization and fitting ability, but also had a local fitting ability, so it could further improve the accuracy of prediction. The calculation equation of the hybrid kernel function is shown as Equation (8).

$$
k_{h y b}=\delta k_{\text {poly }}+(1-\delta) k_{r b f}, 0 \leq \delta \leq 1
$$

where $k_{\text {poly }}$ is polynomial kernel; $k_{r b f}$ is Gaussian kernel; and $\delta$ is weight coefficient.

\subsubsection{Parameter Optimization of SVR Regression Model}

WOA was used to intelligently optimize the penalty factor $c$ and the kernel function parameter $\mathrm{g}$ of the hybrid kernel SVR regression model, and at the same time, adjust the weight coefficient $\delta$ for hybrid kernel optimization [34,35].

The WOA consists of three operators, which realize the optimal search by simulating humpback whales surrounding prey, bubble net attack and prey predation strategy [36-38]. The steps of SVR regression model construction are as follows:

(1) Parameter initialization. Normalize the calibration set and the prediction set, set the number of individuals in the WOA population $N$ and the number of iterations $T E$, and set the optimization range of $c$ and $g$.

(2) Calculate the fitness function value of the initial position of the whale swarm in the WOA, and use the data with the highest evaluation of the fitness function as the current optimal spatial position of the whale swarm.

(3) Calculate the convergence factor $A$ and the swing factor $C$.

(4) Calculate the fitness of each whale, determine the individual whale with the best current fitness and update the spatial position of the whale swarm.

(5) Evaluate the entire whale population again, find the globally optimal whale individual and its positions, compare the fitness corresponding to the whale swarm position vectors before and after updating, determine the next generation of whale swarm positions, and continue the algorithm iteration.

(6) When the number of iterations reaches the set value $T E$, go to step 7 , otherwise go to step 3. 
(7) The output position corresponding to the global optimal whale is the optimal value of $c$ and $g$ in the SVR model. The optimized $c$ and $g$ are used to establish the SVR regression model.

\section{Results}

\subsection{Orthogonal Test on the Effect of HVEF on the Aged Cotton Seed Vigor}

There were 25 treatments and a check $(\mathrm{CK})$ in the orthogonal test, and the test results are shown in Table 2.

Table 2. Orthogonal germination experiment data of aged cotton seeds.

\begin{tabular}{|c|c|c|c|c|c|c|c|c|}
\hline NO. & $\begin{array}{c}\text { HVEF } \\
\text { Intensity } \\
(\mathrm{kV} / \mathrm{cm})\end{array}$ & $\begin{array}{c}\text { Frequency } \\
(\mathbf{H z})\end{array}$ & $\begin{array}{c}\text { Treatment } \\
\text { Time (s) }\end{array}$ & $\begin{array}{c}\text { Germination } \\
\text { Rate } \\
(\%)\end{array}$ & $\begin{array}{c}\text { Germination } \\
\text { Potential } \\
(\%)\end{array}$ & $\underset{\text { Index }}{\text { Germination }}$ & Vigor Index & $\begin{array}{c}\text { Comprehensive } \\
\text { Germination } \\
\text { Index }\end{array}$ \\
\hline CK & 0 & 0 & 0 & $63 \pm 5$ & $57 \pm 2$ & $46 \pm 3$ & $695 \pm 39$ & 232 \\
\hline 1 & 2.4 & 1 & 20 & $74 \pm 5$ & $64 \pm 5$ & $53 \pm 4$ & $805 \pm 56$ & 294 \\
\hline 2 & 2.4 & 5 & 40 & $75 \pm 4$ & $69 \pm 5$ & $55 \pm 3$ & $757 \pm 45$ & 253 \\
\hline 3 & 2.4 & 10 & 60 & $69 \pm 4$ & $63 \pm 2$ & $51 \pm 3$ & $721 \pm 38$ & 241 \\
\hline 4 & 2.4 & 20 & 80 & $84 \pm 5$ & $69 \pm 1$ & $57 \pm 1$ & $862 \pm 21$ & 288 \\
\hline 5 & 2.4 & 50 & 100 & $69 \pm 1$ & $63 \pm 2$ & $50 \pm 1$ & $765 \pm 16$ & 255 \\
\hline 6 & 2.6 & 1 & 40 & $75 \pm 3$ & $71 \pm 2$ & $55 \pm 2$ & $815 \pm 36$ & 272 \\
\hline 7 & 2.6 & 5 & 60 & $71 \pm 2$ & $68 \pm 0$ & $53 \pm 1$ & $711 \pm 15$ & 237 \\
\hline 8 & 2.6 & 10 & 80 & $69 \pm 5$ & $67 \pm 5$ & $57 \pm 4$ & $878 \pm 57$ & 293 \\
\hline 9 & 2.6 & 20 & 100 & $75 \pm 4$ & $63 \pm 4$ & $53 \pm 3$ & $763 \pm 47$ & 255 \\
\hline 10 & 2.6 & 50 & 20 & $75 \pm 2$ & $67 \pm 2$ & $54 \pm 1$ & $883 \pm 11$ & 295 \\
\hline 11 & 3.0 & 1 & 60 & $78 \pm 4$ & $73 \pm 2$ & $58 \pm 3$ & $935 \pm 44$ & 312 \\
\hline 12 & 3.0 & 5 & 80 & $73 \pm 2$ & $69 \pm 2$ & $54 \pm 2$ & $779 \pm 25$ & 260 \\
\hline 13 & 3.0 & 10 & 100 & $75 \pm 2$ & $69 \pm 2$ & $55 \pm 2$ & $830 \pm 29$ & 277 \\
\hline 14 & 3.0 & 20 & 20 & $71 \pm 2$ & $61 \pm 5$ & $51 \pm 3$ & $879 \pm 47$ & 293 \\
\hline 15 & 3.0 & 50 & 40 & $65 \pm 3$ & $58 \pm 7$ & $47 \pm 2$ & $880 \pm 38$ & 294 \\
\hline 16 & 3.4 & 1 & 80 & $72 \pm 8$ & $64 \pm 6$ & $49 \pm 5$ & $774 \pm 66$ & 258 \\
\hline 17 & 3.4 & 5 & 100 & $77 \pm 1$ & $71 \pm 1$ & $56 \pm 1$ & $981 \pm 10$ & 328 \\
\hline 18 & 3.4 & 10 & 20 & $67 \pm 2$ & $60 \pm 2$ & $48 \pm 1$ & $742 \pm 19$ & 248 \\
\hline 19 & 3.4 & 20 & 40 & $65 \pm 2$ & $61 \pm 3$ & $48 \pm 2$ & $817 \pm 28$ & 273 \\
\hline 20 & $\begin{array}{l}3.4 \\
3.4\end{array}$ & 50 & 60 & $78 \pm 3$ & $69 \pm 4$ & $56 \pm 3$ & $944 \pm 46$ & 315 \\
\hline 21 & 3.6 & 1 & 100 & $69 \pm 4$ & $63 \pm 3$ & $51 \pm 2$ & $942 \pm 43$ & 314 \\
\hline 22 & 3.6 & 5 & 20 & $73 \pm 2$ & $66 \pm 2$ & $53 \pm 2$ & $939 \pm 29$ & 314 \\
\hline 23 & 3.6 & 10 & 40 & $69 \pm 1$ & $65 \pm 9$ & $52 \pm 1$ & $934 \pm 26$ & 312 \\
\hline 24 & 3.6 & 20 & 60 & $74 \pm 2$ & $67 \pm 6$ & $54 \pm 1$ & $945 \pm 21$ & 316 \\
\hline 25 & 3.6 & 50 & 80 & $66 \pm 2$ & $62 \pm 3$ & $49 \pm 2$ & $765 \pm 34$ & 255 \\
\hline
\end{tabular}

The HVEF intensity, frequency and treatment time of orthogonal test data were the inputs of SVR model, and the comprehensive germination index was the output of SVR model. In order to improve the prediction accuracy of the SVR model, the raw sample data in Table 1 were normalized, as shown in Table 3.

Table 3. Normalization of the orthogonal experiment data.

\begin{tabular}{ccccc}
\hline NO. & $\begin{array}{c}\text { HVEF Intensity } \\
(\mathbf{k V / c m})\end{array}$ & $\begin{array}{c}\text { Frequency } \\
\mathbf{( H z )}\end{array}$ & $\begin{array}{c}\text { Treatment Time } \\
\mathbf{( s )}\end{array}$ & $\begin{array}{c}\text { Comprehensive } \\
\text { Germination } \\
\text { Index }\end{array}$ \\
\hline CK & 0 & 0 & 0 & 0.69 \\
1 & 0.60 & 0.02 & 0.20 & 0.77 \\
2 & 0.60 & 0.10 & 0.40 & 0.77 \\
3 & 0.60 & 0.20 & 0.60 & 0.77 \\
4 & 0.60 & 0.40 & 0.80 & 0.79 \\
5 & 0.60 & 1.00 & 1.00 & 0.81 \\
6 & 0.65 & 0.02 & 0.40 & 0.78 \\
7 & 0.65 & 0.10 & 0.60 & 0.78 \\
8 & 0.65 & 0.20 & 0.80 & 0.78 \\
9 & 0.65 & 0.40 & 1.00 & 0.79 \\
10 & 0.65 & 1.00 & 0.20 & 0.87 \\
11 & 0.75 & 0.02 & 0.60 & 0.86 \\
12 & 0.75 & 0.10 & 0.80 & 0.83 \\
13 & 0.75 & 0.20 & 1.00 & 0.83 \\
14 & 0.75 & 0.40 & 0.20 & 0.84 \\
\hline
\end{tabular}


Table 3. Cont.

\begin{tabular}{ccccc}
\hline NO. & $\begin{array}{c}\text { HVEF Intensity } \\
(\mathbf{k V / c m})\end{array}$ & $\begin{array}{c}\text { Frequency } \\
\mathbf{( H z )}\end{array}$ & $\begin{array}{c}\text { Treatment Time } \\
\mathbf{( s )}\end{array}$ & $\begin{array}{c}\text { Comprehensive } \\
\text { Germination } \\
\text { Index }\end{array}$ \\
\hline 15 & 0.75 & 1.00 & 0.40 & 0.81 \\
16 & 0.85 & 0.02 & 0.80 & 0.89 \\
17 & 0.85 & 0.10 & 1.00 & 0.90 \\
18 & 0.85 & 0.20 & 0.20 & 0.84 \\
19 & 0.85 & 0.40 & 0.40 & 0.83 \\
20 & 0.85 & 1.00 & 0.60 & 0.80 \\
21 & 0.90 & 0.02 & 1.00 & 0.94 \\
22 & 0.90 & 0.10 & 0.20 & 0.88 \\
23 & 0.90 & 0.20 & 0.40 & 0.85 \\
24 & 0.90 & 0.40 & 0.60 & 0.85 \\
25 & 0.90 & 1.00 & 0.80 & 0.80 \\
\hline
\end{tabular}

\subsection{Kernel Function Selection of SVR}

When weight coefficient $\delta=0$, the kernel used in SVR was Gaussian kernel, and the Gaussian kernel parameters were optimized by WOA. As shown in Figure 2, the optimized penalty factor $c=169.3445$, the kernel function parameter $g=0.022933$, and MSE $=0.035128$ under this condition.

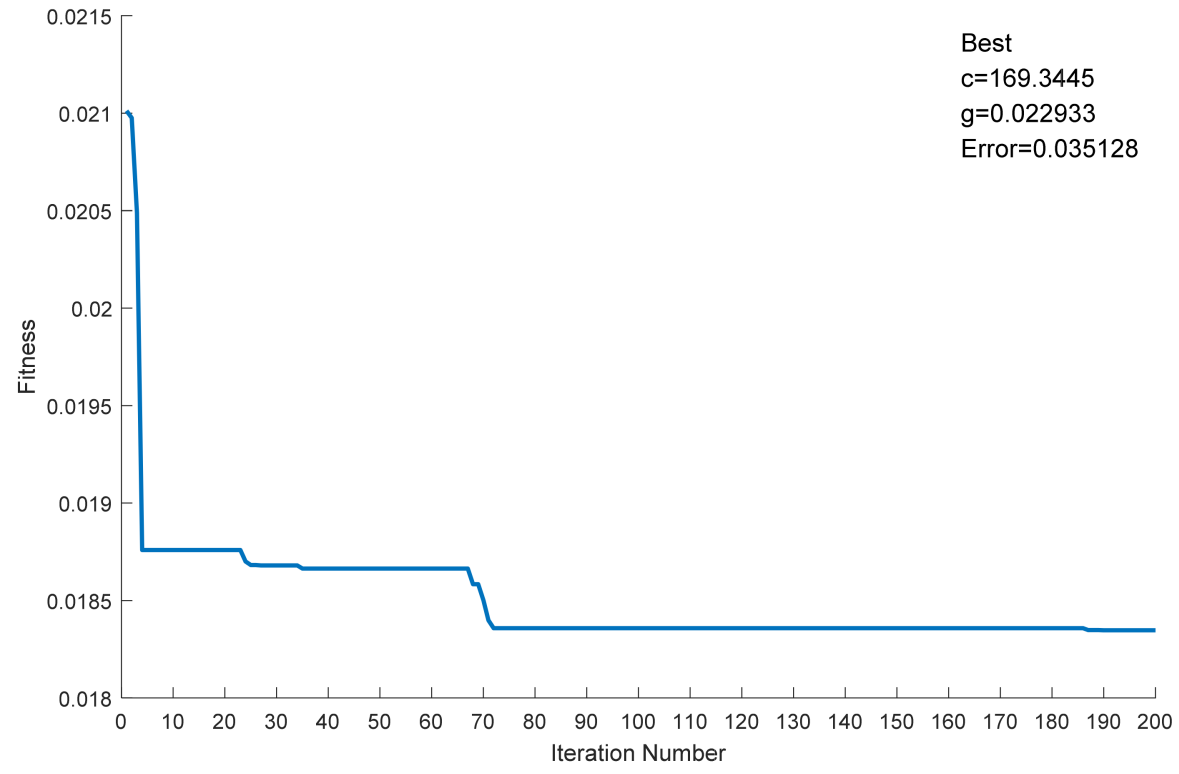

Figure 2. Gaussian kernel parameters optimization.

The weight coefficient of the kernel function was set to the reciprocal of the number of sample features, that is, $\delta=\frac{1}{3}$ in Equation (8). The kernel used in SVR was Gaussian and polynomial hybrid kernel function, then WOA was used to optimize the parameters of the hybrid kernel. As shown in Figure 3, the optimized penalty factor $c=663.7379$, the kernel function parameter $g=0.01133$, and MSE $=0.01835$ under this condition. 


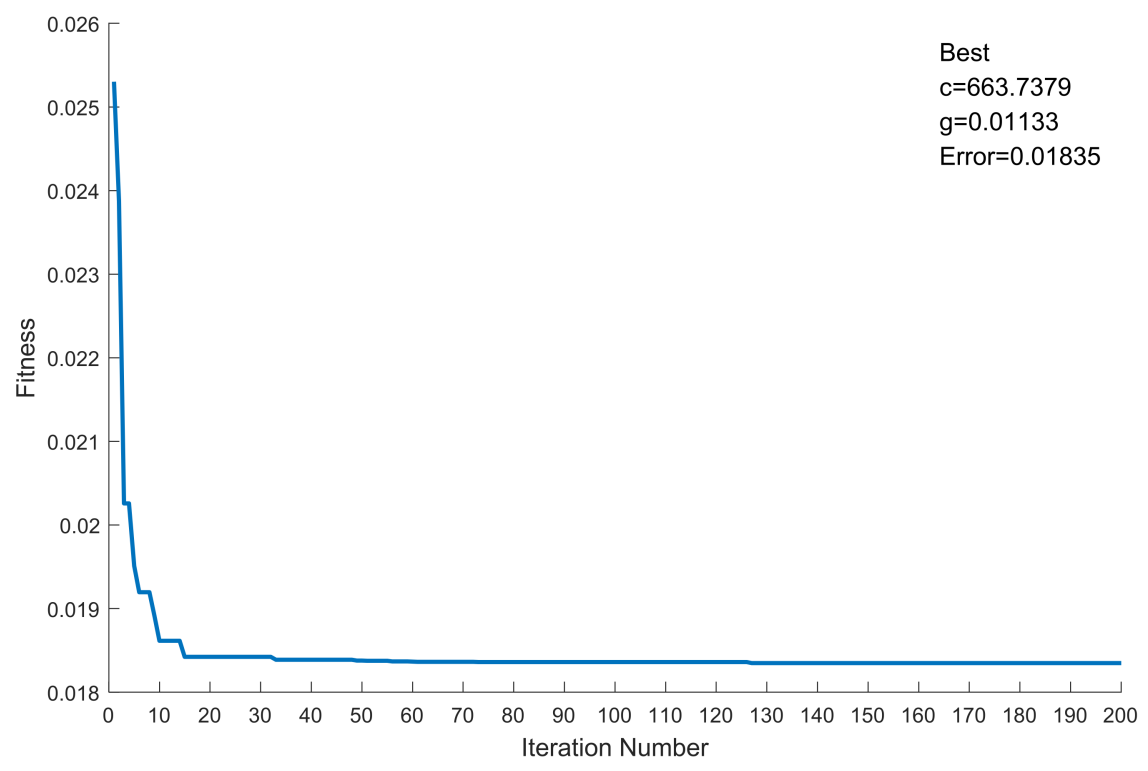

Figure 3. Hybrid kernel parameters optimization.

It can be seen from Figures 2 and 3 that, using WOA to optimize the kernel function parameters of the SVR model, the MSE of the hybrid kernel SVR was smaller than the MSE of the Gaussian kernel SVR, and the penalty factor $c$ after the optimization of the hybrid kernel SVR was significantly greater than that of the Gaussian kernel SVR, so the hybrid kernel was selected as the kernel function of the SVR in this study.

\subsection{Predictive Analysis of Hybrid WOA-SVR}

In further analysis, $80 \%$ of the seed germination orthogonal test data was randomly selected as the training set, and the remaining $20 \%$ as the test set. The optimized hybrid WOA-SVR was used to train the regression model of seed comprehensive germination index. The training results are shown in Figure 4. Then, the WOA-SVR model was used to predict the comprehensive germination index of the training set, and compared with the actual value, the correlation coefficient between the predicted value and the actual value was $R=0.9880$, which showed that there was a high linear positive correlation between the comprehensive germination index predicted by the hybrid WOA-SVR and the actual index.

To verify the generalization performance of the hybrid WOA-SVR of seed comprehensive germination index, the remaining 5 groups of samples (after randomly selecting 20 groups of training sets) were predicted as the test set, and the predicted values of the test set were compared with the actual values, as shown in Figure 5. The correlation coefficient between the predicted value of the test set and the actual value was $R=0.9301$, indicating that on the test set, there was also a high linear positive correlation between the comprehensive germination index of seeds predicted by hybrid WOA-SVR and the actual. The generalization performance of hybrid WOA-SVR of the comprehensive seed germination index was suitable, and it avoided the overfitting problem of SVR training on a small-sample data set. The optimal comprehensive germination index of aged cotton seeds predicted by the hybrid WOA-SVR was 329, and the optimal HVEF dosage to treat aged cotton seeds was $3.64 \mathrm{kV} / \mathrm{cm} \times 99 \mathrm{~s}$, the frequency was $1.4 \mathrm{~Hz}$. 


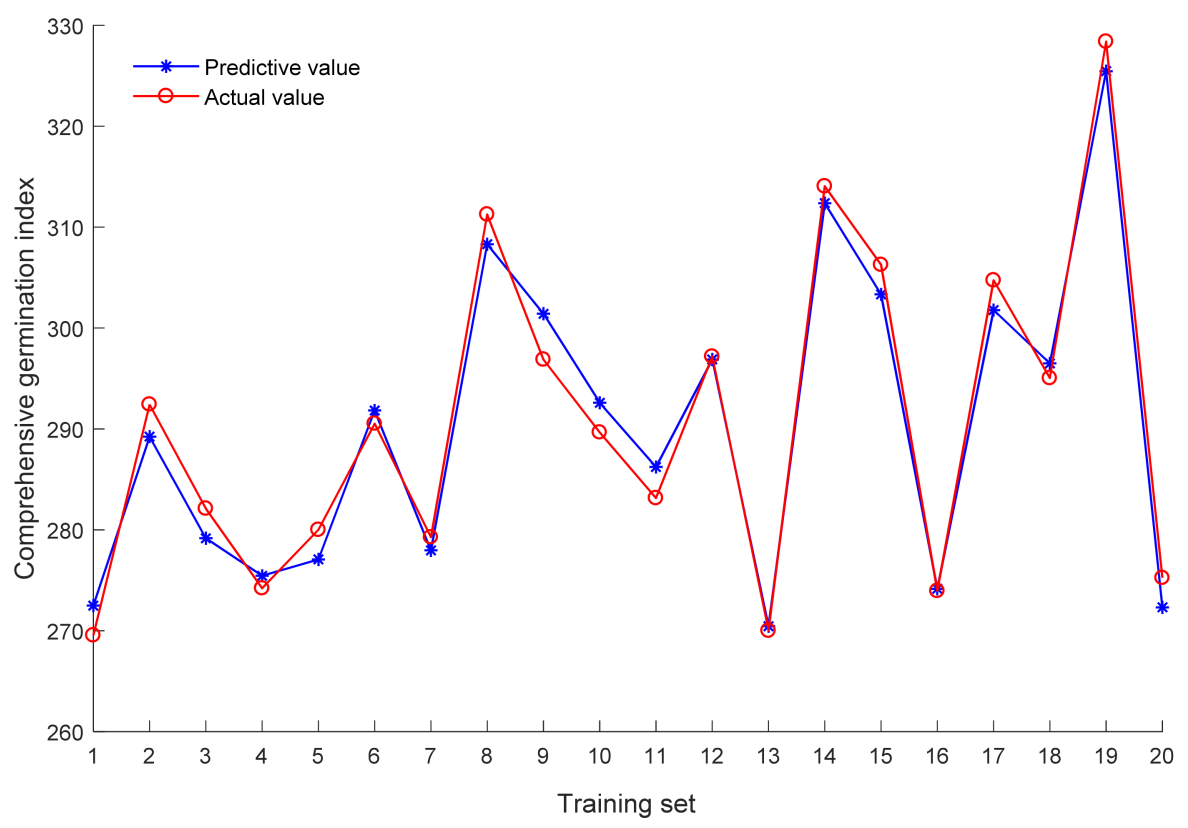

Figure 4. Training results of hybrid WOA-SVR for seed comprehensive germination index.

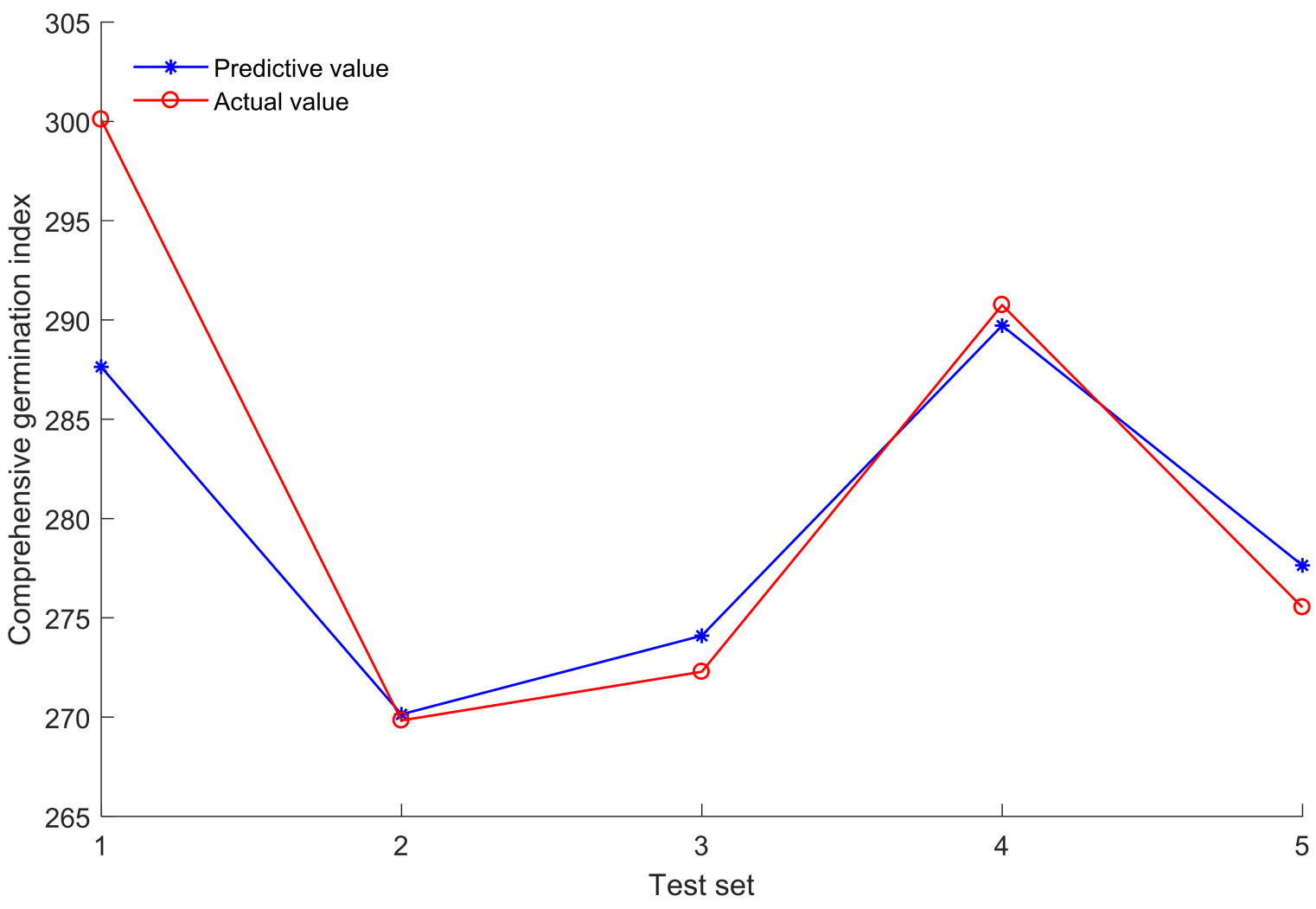

Figure 5. Test results of hybrid WOA-SVR for seed comprehensive germination index.

\subsection{Seed Germination Experimental Verification of WOA-SVR Prediction Parameters}

According to the prediction results of the hybrid WOA-SVR, the optimal electric field intensity for the treatment of aged cotton seeds was $3.64 \mathrm{kV} / \mathrm{cm}$, the treatment time was $99 \mathrm{~s}$, and the frequency was $1.4 \mathrm{~Hz}$; the aged cotton seeds were treated under the conditions and then the seed germination tests were carried out. Comparing with the $\mathrm{CK}$, the results of each index of seed germination are shown in Table 4; here, the germination rate of aged 
cotton seeds increased by $43 \%$, the germination potential increased by $19 \%$, the germination index increased by $29 \%$, the vigor index increased by $48 \%$, the comprehensive germination index increased by $48 \%$, and each index reached a very significant difference $(p<0.01)$. On the other hand, the difference between the comprehensive germination index predicted by WOA-SVR and the experimental was $4 \%$.

Table 4. Verification of Test Results.

\begin{tabular}{|c|c|c|c|c|c|}
\hline $\begin{array}{c}\text { Test } \\
\text { Group }\end{array}$ & $\begin{array}{c}\text { Germination } \\
\text { Rate } \\
(\%)\end{array}$ & $\begin{array}{c}\text { Germination } \\
\text { Potential } \\
(\%)\end{array}$ & $\begin{array}{l}\text { Germination } \\
\text { Index }\end{array}$ & Vigor Index & $\begin{array}{l}\text { Comprehensive } \\
\text { Germination } \\
\text { Index }\end{array}$ \\
\hline $\begin{array}{c}\text { Optimal } \\
\text { HVEF }\end{array}$ & 90 & 67 & 58 & 1029 & 343 \\
\hline $\begin{array}{c}\text { Treatment } \\
\text { CK }\end{array}$ & 63 & 56 & 45 & 695 & 232 \\
\hline
\end{tabular}

\section{Discussion}

The biological effect of the HVEF is the primary physical and chemical reactions in the organism caused by the electric field, thereby forming a comprehensive after-effect $[39,40]$. Appropriate electric field dose (electric field intensity $\times$ treatment time) plays a positive role in promoting seed germination [24,41-43]. Therefore, in order to better improve seed vigor, the HVEF conditions should be optimized. Using the response surface method to optimize the binomial regression model of seed vigor index, the optimal combination of electric field intensity and frequency for HVEF treatment on seeds could be obtained. However, the optimal electric field dose model for seed treatment generally uses the analysis of variance and regression analysis methods based on data statistics, which cannot re-optimize the parameters of the regression model according to the posterior data.

For Tables 2 and 3, the orthogonal test method was used to design the seed treatment with HVEF, and representative experiment samples could be selected from the overall experiment. These representative experimental samples were uniformly dispersed, neat and comparable, which provided effective small-scale sample data sets for SVR model prediction. A simple and feasible method was provided for the selection of appropriate HVEF parameters and treatment time for seed treatment with HVEF. In this study, the mean square error and penalty factor $c$ obtained by using the hybrid kernel SVR were higher than the Gaussian kernel SVR as shown in Figures 2 and 3. Studies had shown that the linear superposition of multiple kernel functions improved the accuracy of the regression prediction model, and had a higher learning ability [44,45], the use of hybrid kernel SVR could further improve the accuracy of prediction. For Figures 4 and 5, the regression model of seed comprehensive germination index was trained through the optimized hybrid kernel SVR, the comprehensive germination index of the training set and the test set was predicted, and the correlation coefficient between the predicted value of the training set and the actual value was 0.9880 , the correlation coefficient between the predicted value of the test set and the actual value was 0.9301 . There was a high linear positive correlation between the seeds comprehensive germination index predicted by the hybrid WOA-SVR model and the actual seed germination index. The hybrid WOA-SVR regression model prediction improved the prediction accuracy and generalization ability of SVR, reduced the overfitting problem of training on small scale sample data sets, and realized accurate global optimization [46].

\section{Conclusions}

In this paper, the electric field intensity, frequency and treatment time of the HVEF were used as the input of the SVR, and the comprehensive seed germination index was used as the predictive output of the SVR. In the study, $80 \%$ of the orthogonal test data of seeds treated with HVEF were randomly selected as the training set and the remaining $20 \%$ as the test set. WOA was used to optimize the kernel function parameters of SVR, and the optimized hybrid WOA-SVR prediction model of seed comprehensive germination index 
was obtained. The seed comprehensive germination index predicted by the optimized WOA-SVR was highly linearly and positively correlated with the actual comprehensive germination index of the aged cotton seeds, which effectively avoided the overfitting problem of SVR training on the small-sample data set, and provided a machine learning method for predicting the optimal electric field dose (electric field strength $\times$ treatment time) of HVEF-treated seeds. The parameters of the WOA-SVR can be re-optimized by using the posterior data of seed germination experiment, which provided a meaningful reference for the parameter optimization of seeds treated by HVEF.

The optimal comprehensive germination index of aged cotton seeds predicted by the hybrid WOA-SVR model was 329, the HVEF intensity was $3.64 \mathrm{kV} / \mathrm{cm}$, the frequency was $1.4 \mathrm{~Hz}$, and the seed treatment time was $99 \mathrm{~s}$; the aged cotton seeds were thus treated for $99 \mathrm{~s}$ in HVEF with an intensity of $3.64 \mathrm{kV} / \mathrm{cm}$ and a frequency of $1.4 \mathrm{~Hz}$. The germination test of the aged cotton seeds treated by the HVEF was carried out and, comparing with CK, the germination rate of the seeds increased by $43 \%$, the germination potential increased by $19 \%$, the germination index increased by $29 \%$, and the vitality index increased by $48 \%$. The actual comprehensive germination index of the aged cotton seeds was 343, which has a deviation of $4 \%$ from the predicted.

Author Contributions: Methodology, J.M.; software, J.M.; validation, F.L. and M.L.; formal analysis, Z.S., J.M. and Y.Y.; data curation, Z.S., J.M. and Y.Y.; writing—original draft preparation, Y.Y.; writingreview and editing, Z.S., J.M. and Y.Y.; visualization, Q.P., B.L. and X.S.; supervision, Q.P., B.L., F.L., M.L. and X.S.; project administration, Y.Y.; funding acquisition, Y.Y. All authors have read and agreed to the published version of the manuscript.

Funding: This research was funded by Shandong Provincial Natural Science Foundation, China, grant number ZR2019MEE065.

Institutional Review Board Statement: Not applicable.

Informed Consent Statement: Informed consent was obtained from all subjects involved in the study.

Data Availability Statement: The authors confirm that the data supporting the findings of this study are available within the article.

Conflicts of Interest: The authors declare no conflict of interest.

\section{References}

1. Mei, H.X.; Liu, Y.Y.; Wu, K.; Du, Z.W.; Zheng, Y.Z. Changes of physiological characteristics and appropriate detection method of seed vigor in aging treatment of sesame seeds. North China J. Agric. 2013, 28, 169-174.

2. Yu, B.; Du, S.G.; Luo, L.P. Determination method of seed vigor. Sci. Sin. Life Sci. 2015, 45, 709-713.

3. Tan, M.; Xu, J.; Li, F.D.; Zhang, C.Q. Physiological mechanisms of improving rice (Oryza sativa L.) seed vigor through arc-toothshaped corona discharge field treatment. Aust. J. Crop Sci. 2014, 8, 1495-1502.

4. Tan, M. Research of Effects and Physiology Mechanisms on Rice Seed Vigor with Corona Discharge Field Treatment. Master's Dissertation, Shandong Agricultural University, Taian, China, 2014; pp. 1-48.

5. Cai, X.W.; Wang, B. Study on the Biological Domino Effect on Eggplant Seeds in High Voltage Static Electricity Field. Seed 2003, 1, 19-20.

6. Cakmak, T.; Cakmak, Z.E.; Dumlupinar, R.; Tekinay, T. Analysis of apoplastic and symplastic antioxidant system in shallot leaves: Impacts of weak static electric and magnetic field. J. Plant Physiol. 2012, 169, 1066-1073. [CrossRef] [PubMed]

7. Liao, D.Q.; Zhang, H.L.; Li, Z.C.; John, B. Characterization of Evolution and Tissue-Expression of Rice (Oryza sativa L.) Amylase Genes. Acta Agron. Sin. 2010, 1, 17-27. [CrossRef]

8. Stanley, D.; Famden, K.J.F.; Macrae, E.A. Plant $\alpha$-amylases: Functions and roles in carbohydrate metabolism. Biol.-Sect. Cell. Mol. Biol. 2005, 16, 65-71.

9. Katarzyna, D.; Petr, D.; Valentina, P.; Antonio, A.V.; Lars, W.; Christine, F.; Federico, G.G. Effect of pulsed electric field on the germination of barley seeds. LWT-Food Sci. Technol. 2012, 47, 161-166.

10. Yan, Y.F.; Zhou, S.X.; Song, Z.H.; Li, F.D.; Zhang, C.Q.; Zhang, X.H.; Song, H.L. Effects of frequency and voltage of high voltage pulsed electric field on improving vigor of aged cotton seed. Trans. Chin. Soc. Agric. Eng. 2017, 33, 310-314.

11. Xu, J.; Tan, M.; Guo, S.Z.; Yan, Y.F.; Song, H.L.; Zhang, C.Q.; Li, F.D. Difference of three kinds of corona discharge fields in processing paddy seeds. Trans. ASABE 2014, 57, 1729-1739.

12. Xu, J.; Guo, S.Z.; Jia, G.L.; Zhang, N.; Song, Z.H.; Xu, G.H.; Zhang, C.Q.; Li, F.D. Effect of corona discharge field processing on vigor of three layers of paddy seeds. Trans. Chin. Soc. Agric. Eng. (Trans. CSAE) 2015, 31 (Suppl. 1), 307-314. 
13. Lynikiene, S.; Pozeliene, A.; Rutkauskas, G. Influence of corona discharge field on seed viability and dynamics of germination. Int. Agrophysics 2006, 20, 195-200.

14. Marček, T.; Kovač, T.; Jukić, K.; Lončarić, A.; Ižaković, M. Application of high voltage electrical discharge treatment to improve wheat germination and early growth under drought and salinity conditions. Plants 2021, 10, 2137. [CrossRef] [PubMed]

15. Huang, H.Y.; Du, N.; Han, Z. Effects of wheat seedling cold resistance through pretreatments of seeds with high voltage electrostatic field. Seed 2016, 8, 24-26.

16. Yang, T.Q.; Zhu, H.Y.; Hua, H.G.; Liu, Y.; Hu, Y.F.; Wang, Z. Sensitivity to drought stress of the germination and seeding growth of caragana korshinskii from seeds treated with electric field. J. Desert Res. 2013, 33, 1717-1722.

17. Zhong, J.N. The Effect of High-Voltage Prick Electrostatic Field on Saline-Alkali Stress Resistance of Leymus Chinensis Seedling. Master's Dissertation, Northeast Normal University, Changchun, China, 2007; pp. 1-44.

18. Lv, J.G.; Yang, T.Q.; Su, E.G.; Deng, Y.B.; Na, R. Effect of electric field treatment of wheat seeds on the salt resistance of growth in seedling stage. Acta Sci. Nat. Univ. NeiMongol 2001, 32, 707-710.

19. Bormashenko, E.; Shapira, Y.; Grynyov, R.; Whyman, G.; Bormashenko, Y.; Drori, E. Interaction of cold radiofrequency plasma with seeds of beans (Phaseolus vulgaris). J. Exp. Bot. 2015, 66, 4013-4021. [CrossRef] [PubMed]

20. Wang, J.; Song, H.; Song, Z.; Lu, Y.; Yan, Y.; Li, F. Effect of Positive and Negative Corona Discharge Field on Vigor of Millet Seeds IEEE Access 2020, 8, 50268-50275. [CrossRef]

21. Patwardhan, M.S.; Gandhare, W.Z. High voltage electric field effects on the germination rate of tomato seeds. Acta Agrophys. 2013, 20, 403-413.

22. Bao, S.S.G.W.; Ma, Z.X. Correlation Between Crop Seed Growth and Electric Field Condition. Chin. Bull. Bot. $2010,45,384-391$.

23. Yan, Y.F.; Han, S.Q.; Zhou, S.X.; Song, Z.H.; Li, F.D.; Zhang, C.Q.; Zhang, X.H.; Wang, J. Parameter optimization of extremely low frequency and high voltage pulsed electric field to improve aged cotton seed vigor. Trans. Chin. Soc. Agric. Eng. 2017, 33, 301-307.

24. Chen, J.Z.; Hu, J.F.; Du, H.L.; Wang, H.J.; Wang, Y.S. Response of endogenous hormone in germination of millet seeds to optimal high voltage electrostatic field. Trans. Chin. Soc. Agric. Eng. 2016, 32, 286-292.

25. Xiao, Z.T. Development of Grain Moisture Detection Instrument Based on Near Infrared Spectroscopy. Master's Dissertation, Nanchang Hangkong University, Nanchang, China, 2018; pp. 1-79.

26. Lin, B.Y. Study on Prediction of Somatic Cell Count in Raw Milk and Inflammation Grade of Dairy Cattle Based on Dielectric Spectrum. Master's Dissertation, Northwest A\&F University, Yangling, China, 2017; pp. 1-65.

27. Chapelle, O.; Vapnik, V.; Bousquet, O.; Mukherjee, S. Choosing multiple parameters for support vector machines. Mach. Learn. 2002, 46, 131-159. [CrossRef]

28. Sun, J.; Mo, Y.N.; Dai, C.X.; Chen, Y.; Yang, N.; Tang, Y. Detection of moisture content of tomato leaves based on dielectric properties and IRIV-GWO-SVR algorithm. Trans. Chin. Soc. Agric. Eng. 2018, 34, 188-195.

29. Mirjalili, S.; Lewis, A. The whale optimization algorithm. Adv. Eng. Softw. 2016, 95, 51-67. [CrossRef]

30. Na, R.; Yang, S.; Xing, W.J.; Yang, T.Q. Effect of fields radiation on callus inductivity and differentiation of Hedysarum scoparium Fisch seed. J. Nucl. Agric. Sci. 2010, 24, 748-751.

31. Zhou, S.X. Effects of High-Pressure Uniform Electric Field and Pulsed Electric Field on Cotton Seed Vigor. Master's Dissertation, Shandong Agricultural University, Taian, China, 2017; pp. 1-51.

32. Zhou, Y.R.; Cui, D.W. Prediction of annual runoff based on hybrid nuclear shts-svm. J. Water Resour. Water Eng. $2019,30,66-72$.

33. Zheng, M.M. Research on Hybrid Kernel Function Based on Support Vector Machine. Master's Dissertation, East China Normal University, Wuhan, China, 2019; pp. 1-70.

34. Liu, H.Y.; Zhen, C.G. WOA-LSSVM basedboiler NOx emission prediction model. J. North China Electr. Power Univ. (Nat. Sci.) 2019, 46, 84-91.

35. Zhang, H.L.; Lv, J.G.; Cheng, L. Parameter Adjustment for Induction Drive System Controller of an Electric Vehicle on WOA. J. Shandong Agric. Univ. 2019, 50, 163-166.

36. Diop, L.; Samadianfard, S.; Bodian, A.; Yaseen, Z.A.; Ghorbani, M.A.; Salimi, H. Annual rainfall forecasting using hybrid artificial intelligence model: Integration of multilayer perceptron with whale optimization algorithm. Water Resour. Manag. 2020, 34, 733-746. [CrossRef]

37. Babak, M.; Saeid, M. Modeling daily reference evapotranspiration via a novel approach based on support vector regression coupled with whale optimization algorithm. Agric. Water Manag. 2020, 237, 106145-106157.

38. Song, H.l.; Yan, Y.F.; Song, Z.H.; Sun, J.X.; Li, Y.D.; Li, F.D. Nondestructive testing model for maize grain moisture content established by screening dielectric parameters and variables. Trans. Chin. Soc. Agric. Eng. 2019, 35, 262-272.

39. Xie, J.F.; Yi, W.S.; Xiong, G.; Zhang, B.S.; Chen, Z.Y. The effect of high electrostatic field on plant cellular transmembrane voltage and micro principles. J. Hubei Univ. (Nat. Sci. Ed.) 2002, 24, 235-237.

40. Harrison, S.L.; Barbosacanovas, G.V.; Swanson, B.G. Saccharomyces cerevisiae structural changes induced by pulsed electric field treatment. LWT-Food Sci. Technol. 1997, 30, 236-240. [CrossRef]

41. Bai, Y.X.; Hu, Y.C. Original mechanism of biological effects of electrostatic field on crop seeds. Trans. Chin. Soc. Agric. Eng. 2003, 19, 49-51.

42. Zhang, B.H.; Li, C.H.; Wang, D.Y. Calculation and test of electric polarization force for rice seed dielectric separation. Trans. Chin. Soc. Agric. Eng. 2012, 28, 96-100. 
43. Zhang, L.; Shen, X.Y.; Yang, F. Influence of high voltage electrostatic field to the biological effect. J. Northeast Agric. Univ. 2000, 31, 307-312.

44. Chen, B.; Zheng, K.L.; Wang, J.H. Research on multiple-kernel support vector regression method. Intell. Comput. Appl. 2019, 9, 188-191.

45. Liu, Y.Z.; Lai, H.R.; Zhang, D.W.; Liu, F.P.; Jiang, X.L.; Cao, Q.A. Change detection of high resolution remote sensing image alteration based on multi-feature mixed kernel SVM model. Remote Sens. Land Resour. 2019, 31, 16-21.

46. Liang, Y.D.; Luan, Y.C.; Liu, F.Y.; Ji, Z.L.; Zhuang, Y. Forecast of dam deformation by hybrid kernel function least squares support vector machine with improved adaptive particle swarm optimization. Sci. Technol. Eng. 2021, 21, 47-52. 